\title{
A broad spectrum of posterior reversible encephalopathy syndrome - a case series with clinical and paraclinical characterisation, and histopathological findings
}

Fatme Seval Ismail ${ }^{1 *}$, Johannes van de Nes ${ }^{2}$ and Ilka Kleffner ${ }^{1}$

\begin{abstract}
Background: Posterior reversible encephalopathy syndrome (PRES) is clinical-neuroradiologically defined and potentially reversible, so there are limited data about histopathological findings. We aimed to describe the clinical and paraclinical features of patients with PRES with regard to its reversibility.

Methods: This retrospective case series encompasses 15 PRES cases out of 1300 evaluated patients from a single German center between January 1, 2010, and June 15, 2020. PRES was established according to the diagnostic criteria as proposed by the Berlin PRES Study 2012. One of the cases studied was subject to brain autopsy.

Results: From the 15 patients studied (median age 53 years, range 17-73; 11 female), 67\% presented with epileptic seizures, $40 \%$ suffered from encephalopathy with reduced consciousness and $53 \%$ developed delirium, while $47 \%$ had headache and visual disturbances. Subcortical brain MRI abnormalities related to PRES were observed in all patients. One patient developed spinal ischemia and another Guillain-Barré syndrome in addition to PRES. Hypertensive blood pressure was the main underlying/trigger condition in all patients. Clinical symptoms and MRI changes were not reversible in $42 \%$, even progressive in 3 out of these 5 patients. Median time from symptom onset to diagnosis in these non-reversible cases was 7 days (range $0-13$ ), while the median delay in diagnosis in the reversible group was 1 day (range 0-3). Cerebellar/brain stem involvement and status epilepticus were more frequently in patients with non-reversible disease course. Mortality due to PRES occurred in 13\% of these patients. Neuropathological examination of the brain of a 57-year-old female patient revealed major leukencephalopathic changes, fibrinoid necrosis of endothelial cells and fresh petechial hemorrhages in accordance with PRES.
\end{abstract}

Conclusions: Our case series demonstrates that PRES was not reversible in $42 \%$ of the studied patients. Delay in diagnosis seems to contribute to limited reversibility and poor outcome.

Keywords: Posterior reversible encephalopathy syndrome (PRES), Reversibility, Histopathological findings

*Correspondence: FatmeSeval.Ismail@kk-bochum.de; ismaf88@gmail.com ${ }^{1}$ Department of Neurology, University Hospital

Knappschaftskrankenhaus, Ruhr University Bochum, In der Schornau 23-25, 44892 Bochum, Germany

Full list of author information is available at the end of the article

\section{Background}

Posterior reversible encephalopathy syndrome (PRES) is clinical-neuroradiologically defined and potentially reversible [1]. It is pathogenetically characterized by acute cerebral endotheliopathy with failure of the cerebral autoregulation and disruption of the blood-brain barrier leading to vasogenic edema, mostly associated 
with hypertension [1,2]. Cytotoxic edema is reported in a subset of PRES patients [3]. The clinical syndrome of PRES may show a variable spectrum of symptoms that usually occur monophasically, but recurrence in rare cases has been described [4]. Atypical distribution patterns of edema in neuroimaging are possible. Because of a low mortality with 3-6\% [5], the numbers of studies in the literature with histological findings are limited $[2,6$, 7]. Many aspects of this syndrome and the pathogenetic mechanism underlying the development of PRES are as yet incompletely understood [8].

The aim of the study presented here was to describe the spectrum of clinical and paraclinical features (including MRI, CSF and EEG) of patients with PRES with regard to its reversibility. In addition, we report on the neuropathological changes in the brain of a 57-year-old female PRES patient.

\section{Methods}

In this retrospective case series, we identified 15 patients from a total of 1300 evaluated patients using the International Classification of Diseases 10th Revision (ICD-10) diagnostic coding (G93.4, G93.6, I67.4, I67.88) in a single German neurologic center between January 1, 2010, and June 15, 2020. The included 15 patients carried the clinical-neuroradiological diagnosis of PRES according to the diagnostic criteria as proposed by Berlin PRES Study 2012 [1], one of them confirmed on histological examination at autopsy:

(1) Acute development of clinical signs and symptoms typical for PRES, i.e., epileptic seizures, visual abnormalities, headaches, nausea or vomiting and other focal deficits.

(2) Imaging signs typical of PRES, i.e., foci of vasogenic edema in variable distribution and severity with or without foci of restricted diffusion or hemorrhagic manifestations

(3) Presence of often multiple, toxic associations with possible endotheliotoxic effects and/or arterial hypertension (mild to severe degree).

(4) Alternative causes were excluded.

Information on demographic data, clinical presentation, co-morbidities, brain MRI and CT findings, CSF analysis data, EEG findings, anticonvulsive treatment, intensive care treatment, intravenous blood pressure medication as well as maximal systolic blood pressure was obtained from the medical records. Follow-up imaging and clinical information were evaluated for reversibility, graded as complete, incomplete or progressive, while outcome was determined by functional status, as quantified by use of the modified Rankin Scale (mRS) (0-6) at admission and at discharge. In addition, brain autopsy was performed on one of the patients studied.

All procedures/protocols were in accordance with the ethic guidelines of the Ruhr University Bochum. All methods were carried out in accordance with relevant guidelines and regulations. According to legislative regulations in Germany, North Rhine-Westphalia and after consultation of the Institutional Review Board, Medical Faculty of the Ruhr University Bochum, the need for ethical approval and consent is deemed unnecessary for this retrospective case series with data collected during routine clinical work. Approval for brain autopsy was obtained.

\section{Results \\ Clinical and paraclinical findings}

Fifteen patients were identified as clinico-radiologically consistent with PRES, 7 with reversibility (\#1-7), 5 with a non-reversible outcome (\#8-12) and 3 with lack of data on reversibility due to loss of follow-up after referral to another hospital (\#13-15). For detailed information on clinical findings, see Table 1. Eleven patients were female (73\%), median age of all patients was 53 years (range 17-73). The most common neurological symptoms were epileptic seizures occurring in 10 patients (67\%). Four out of these patients also developed status epilepticus, two patients with convulsive status, one patient with convulsive and later non-convulsive status, and another one with non-convulsive status. Impaired vision was reported in 7 patients ( $47 \%$ ), including one with bilateral loss of vision and another one with cortical blindness. Six patients (40\%) suffered from encephalopathy with reduced consciousness, and 6 patients (40\%) developed delirium, while 7 had headache. Mnestic deficits were described in 1 patient, focal neurological deficits (paraplegia, cranial nerve palsy) were found in 2 patients. One patient with myelodysplastic syndrome and secondary acute myeloid leukemia developed PRES and additionally Guillain-Barré syndrome after chemotherapy with allogeneic stem cell transplantation (\#2), another patient with a previous medical history of prostate adenocarcinoma and multiple myeloma developed spinal ischemia in the course of PRES (\#6).

Brain MRIs were available from all patients. Subcortical MRI abnormalities with T2-/FLAIR-hyperintense signal related to PRES were detected in order of decreasing numbers in parieto-occipital lobes (100\%), in temporal lobe and cerebellar hemisphere (each $47 \%)$, in frontal lobe (40\%), brainstem (20\%), basal ganglia (13\%) and in thalamus (7\%). Bilateral involvement was observed in all cases, cortical involvement was described in $47 \%$. Foci of restricted diffusion either in the white matter or in the cortex occurred 


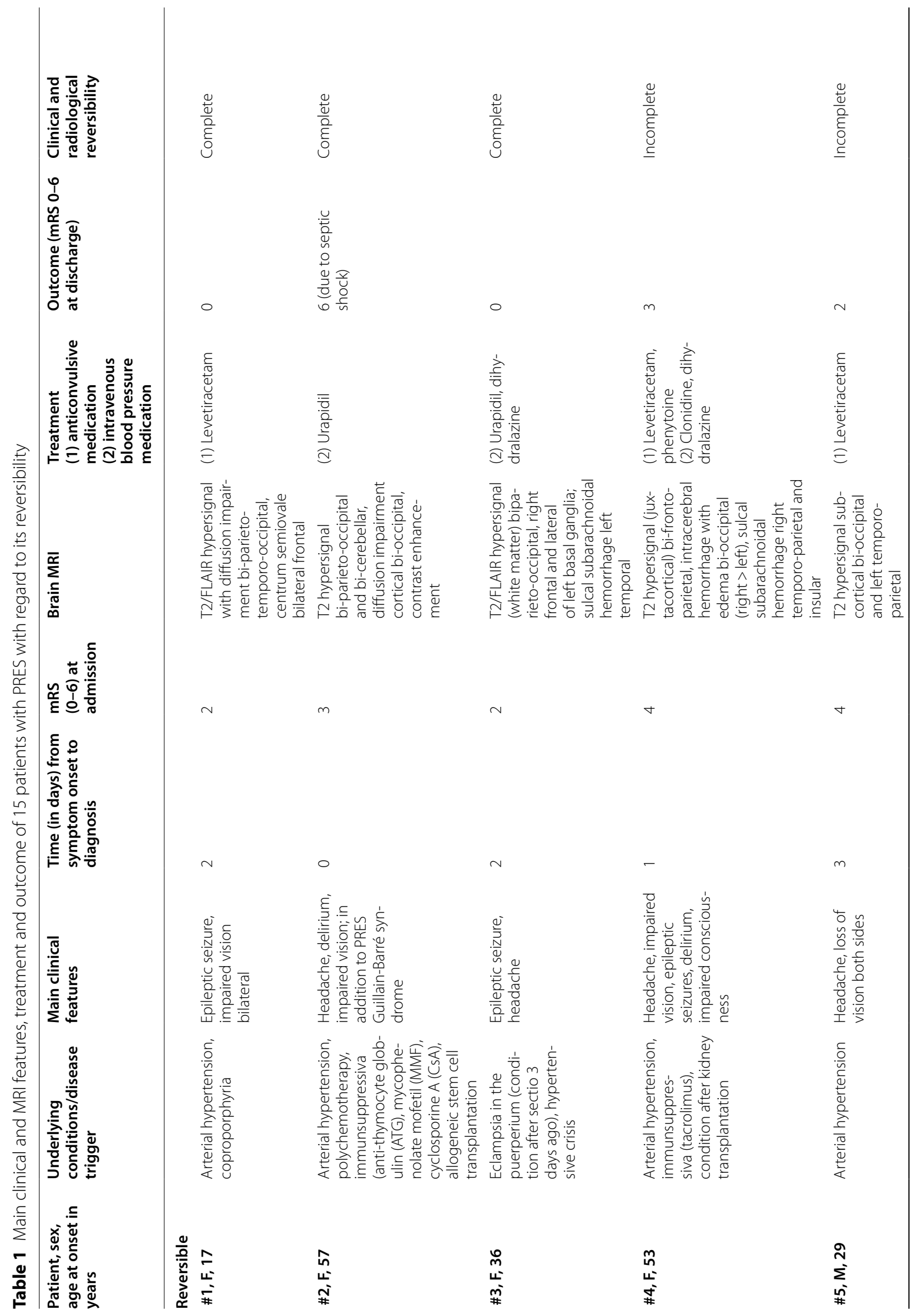




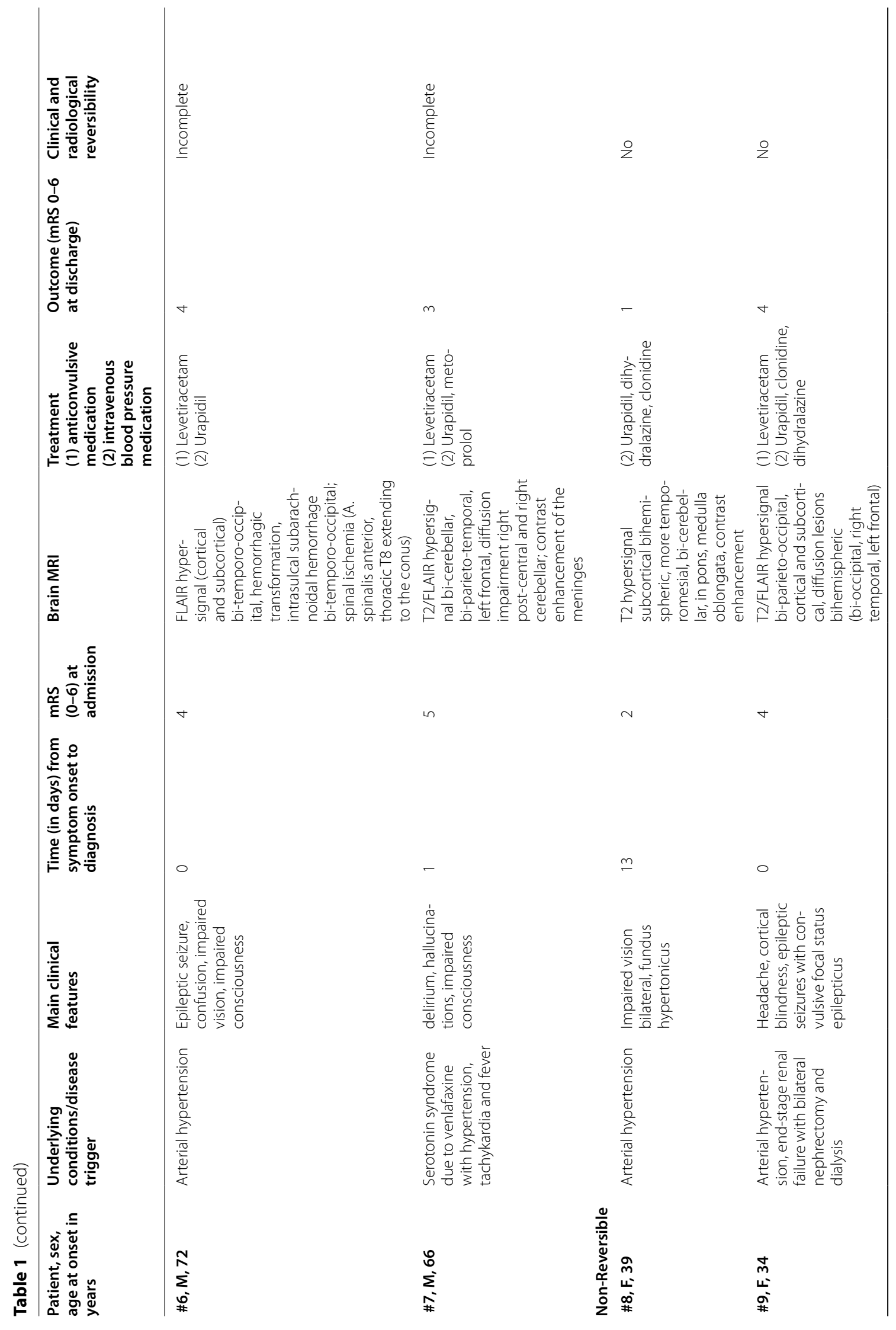




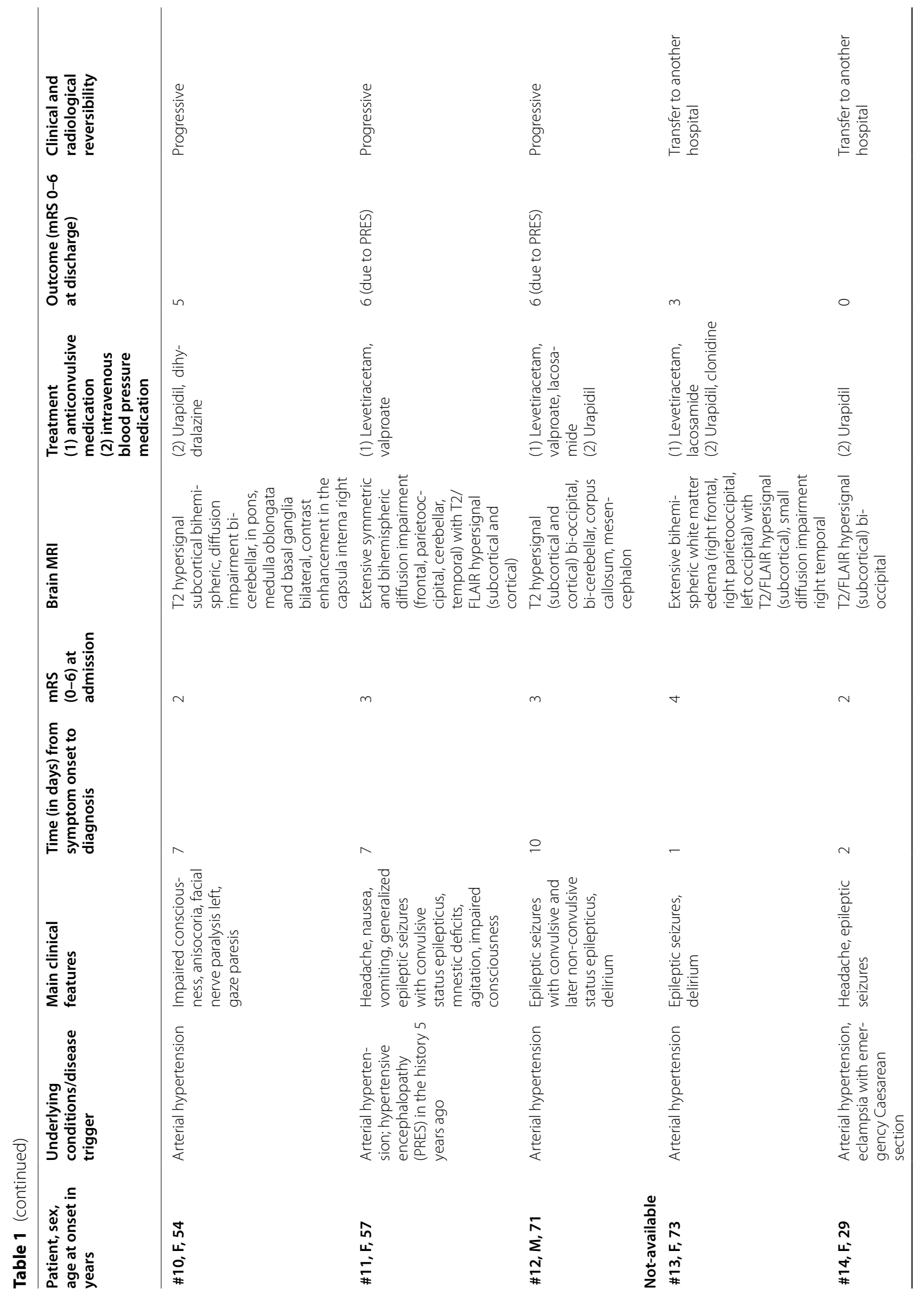




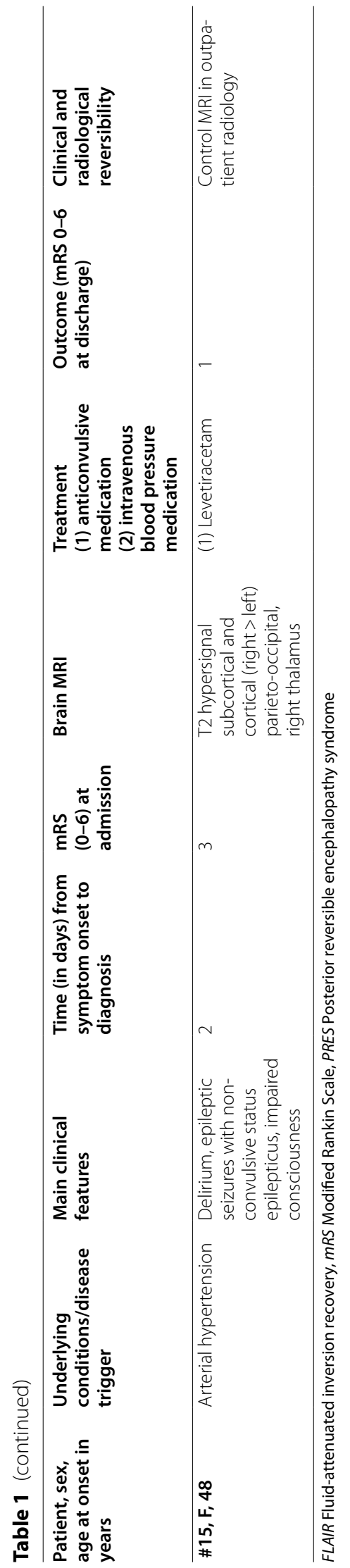


in $47 \%$. Four patients showed contrast enhancement, while 3 patients displayed an intrasulcal subarachnoidal hemorrhage, one of these with an additional intracerebral bleeding. In one patient PET-CT scanning revealed no uptake in brain lesions. For detailed information on neuroradiological findings, see Table 1. In our patients, there was no evidence of vasoconstriction on vascular imaging including MR-angiography, CT-angiography, digital subtraction angiography (DSA) and extra- and transcranial Doppler and Duplex sonography of brain-supplying arteries. MR-angiography alone was performed in $n=4$ patients, in combination with CT-angiography in $n=1$ patient, with Doppler/Duplex sonography in $n=3$ patients, with CT-angiography and Doppler/Duplex sonography in $n=2$ patients. Two patients have received only CTangiography and one in combination with Doppler/ Duplex sonography. DSA was performed in $n=2$ patients.

Cerebral spinal fluid (CSF) analysis was performed in 9 patients (60\%). CSF pleocytosis (median 133 cells $/ \mu$ l, range 15-209) was found in 3/9 patients (33\%) (\#4, 7, 10) with severe disease course of PRES, while elevated protein (median 104, range 69-261 $\mathrm{mg} / \mathrm{dl}$ ) was measured in 5 patients $(56 \%)(\# 4,7-8,12,15)$. Infectious pathogens in CSF were not detected.

EEG was performed in all but one PRES patient (93\%). Epileptiform discharges were detected in 7 patients $(50 \%)$, focal slowing in $7(50 \%)$ and diffuse slowing in 5 patients (36\%).

Underlying conditions and trigger of PRES:

Hypertensive blood pressure was measured at hospital admission in all patients. The maximum systolic blood pressure ranged from 155 to $275 \mathrm{mmHg}$ (median $196 \mathrm{mmHg}$ ). Underlying conditions triggering or contributing to PRES were coproporphyria (\#1), immunosuppressive treatment (tacrolimus (\#4); anti-thymocyte globulin, mycophenolate mofetil, cyclosporine A (\#2)), chemotherapy with allogeneic stem cell transplantation (\#2), eclampsia during pregnancy/puerperium (\#3 and $\# 14$, respectively), serotonine syndrome due to venlafaxine (\#7), history of PRES (\#11) (Table 1).

Comorbidities included tumor (prostate adenocarcinoma $n=1$, multiple myeloma $n=1$, myelodysplastic syndrome with secondary acute myeloid leukemia $n=1)$, type 2 diabetes $(n=3)$, hypertensive nephropathy with glomerular proteinuria $(n=1)$, chronic renal insufficiency $(n=1)$, condition after kidney transplantation $(n=1)$, condition after bilateral nephrectomy with dialysis $(n=1)$, hepatitis of unknown origin $(n=1)$, alcohol dependency $(n=3)$, sepsis $(n=1)$, psychosis $(n=1)$, history of epilepsy due to tuberous sclerosis complex $(n=1)$, and vascular dementia $(n=1)$.

\section{Treatment}

Anticonvulsive treatment with levetiracetam was administered in 10 patients. Intensive care treatment was indicated in 12 cases $(80 \%)$ with median duration of 5 days (range 2-35). Intravenous blood pressure medication as monotherapy or combination (urapidil, clonidine, dihydralazine) was administered in 11 patients (73\%). In one patient with fatal course, corticosteroids were administered before admission possibly contributing to the worsening of PRES due to increased risk of acute hypertension.

\section{Reversibility and outcome}

Complete clinical and brain imaging reversibility was reported in 3 patients (25\%) (\#1-3), incomplete in 4 patients (33\%) (\#4-7). Clinical symptoms and MRI changes were not reversible or even progressive in 5 patients (42\%) (\#8-12) (Table 1). Duration of followup in the 12 patients for whom this was available was median 18,5 days (range 7-141). In the group with nonreversible disease course, median time from symptom onset to diagnosis was 7 days (range $0-13$ ), thus generally longer compared to the group of patients with reversible course (1 day, range $0-3$ ). Cerebellar and brain stem involvement in brain MRI was observed in 4 out of 5 patients with a non-reversible course (80\%) and in 2 out of 7 patients with reversible course (29\%). Status epilepticus was observed only in patients with non-reversible disease course.

Median mRS at admission was 3 (range 2-5) and at discharge 3 (range 0-6). Two patients (13\%) died of PRES, one of those patients with a fatal course was admitted to our department because of status epilepticus and massive brain edema (\#12).

\section{Case \#11 \\ Clinical presentation and neuroradiological examination}

The second patient with a fatal course, a 57-year-old woman (\#11), was known for diabetes type 2 and hypertensive encephalopathy (PRES) in the previous medical history 5 years before. She was referred from another clinic to our hospital for a bifrontal craniotomy because of rapidly progressive generalized brain edema. Immunotherapy including intravenous methylprednisolone for 5 days was carried out for a suspected autoimmune encephalitis in the other clinic before transfer to our hospital. Post-operative MRI revealed bilateral symmetric and extensive diffuse restriction with $\mathrm{T} 2$ hyperintense signaling (frontal, parietooccipital, cerebellar, temporal) as full-blown manifestation of PRES (Fig. 1). Despite intensive medical treatment, the patient eventually died due to supra- and infratentorial herniation. Approval for brain autopsy was obtained. 

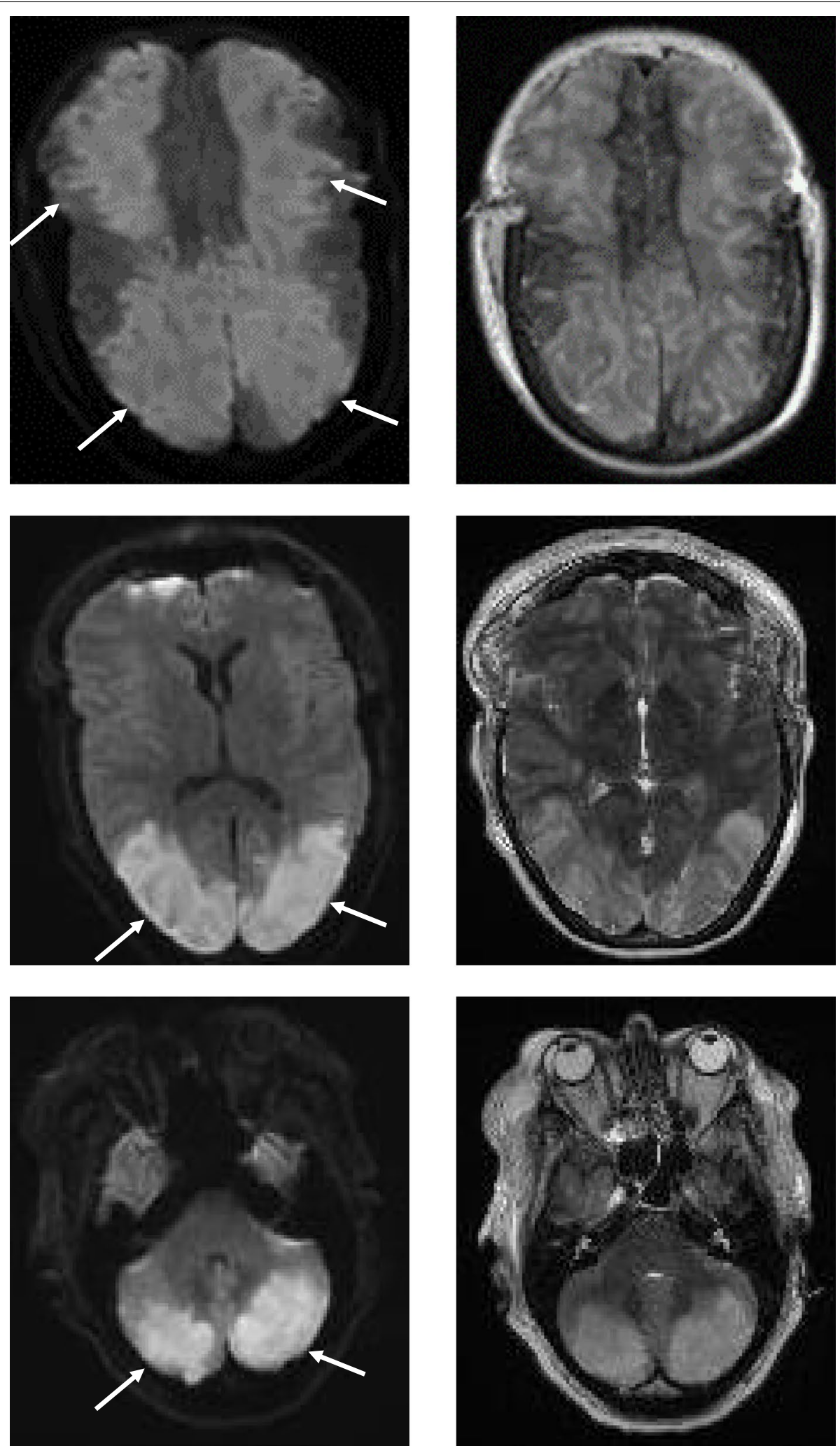

Fig. 1 Brain MRI (transversal sequences) in a fatal case of PRES due to hypertension. Bilateral, symmetric and extensive diffusion restrictions (on diffusion-weighted imaging (DWI)) (white arrows) (left panels) and T2 hyperintense signal (right panels) due to full-blown manifestation of PRES with following bifrontal craniotomy 


\section{Macroscopic examination}

The brain $(1.500 \mathrm{~g})$ showed a generalized edema with flattened brain surface, bilateral uncal and cerebellar tonsillar herniation with major infarctions in the occipital lobes due to compression of the posterior cerebral arteries and in the cerebellar hemispheres due to compression of the superior cerebellar arteries. In addition, a major bifrontal extracalvarian herniation (fungus cerebri) related to bifrontal craniotomy as neurosurgical decompression therapy and major bifrontal infarctions were present (not shown).

\section{Histopathological examination}

Tissue specimens displayed extensive leukencephalopathic changes in a major part of the cerebral and cerebellar hemispheres, viz., diffuse myelin pallor and mild astroglial activation, some thin-walled blood vessels with fibrinoid wall necrosis and minor fresh petechial hemorrhages. In addition, a few macrophages with vacuolated cytoplasm around some blood vessels were observed, indicative of phagocytosis of myelin sheath break down products. The cerebral and cerebellar cortex, basal ganglia and brain stem nuclei showed major hypoxic-ischaemic neuronal changes (Fig. 2A-D).

\section{Discussion}

In this retrospective study on 15 patients with PRES, hypertensive blood pressure from mild to severe degree was detected in all patients, supporting the assumed association of hypertension with the development of PRES. Edematous bilateral lesions were encountered in the occipital and parietal lobes of all patients studied, while additional cerebellar, temporal and frontal lobe involvement occurred in about $50 \%$ of the cases. Involvement of other brain regions was also observed, viz., brain stem (20\%), basal ganglia (13\%) or thalamus (7\%). Hemorrhage and contrast enhancement occurred in some cases (27\%). The most common neurological symptoms were seizures. Visual disturbances, encephalopathy, delirium and headache represented other common

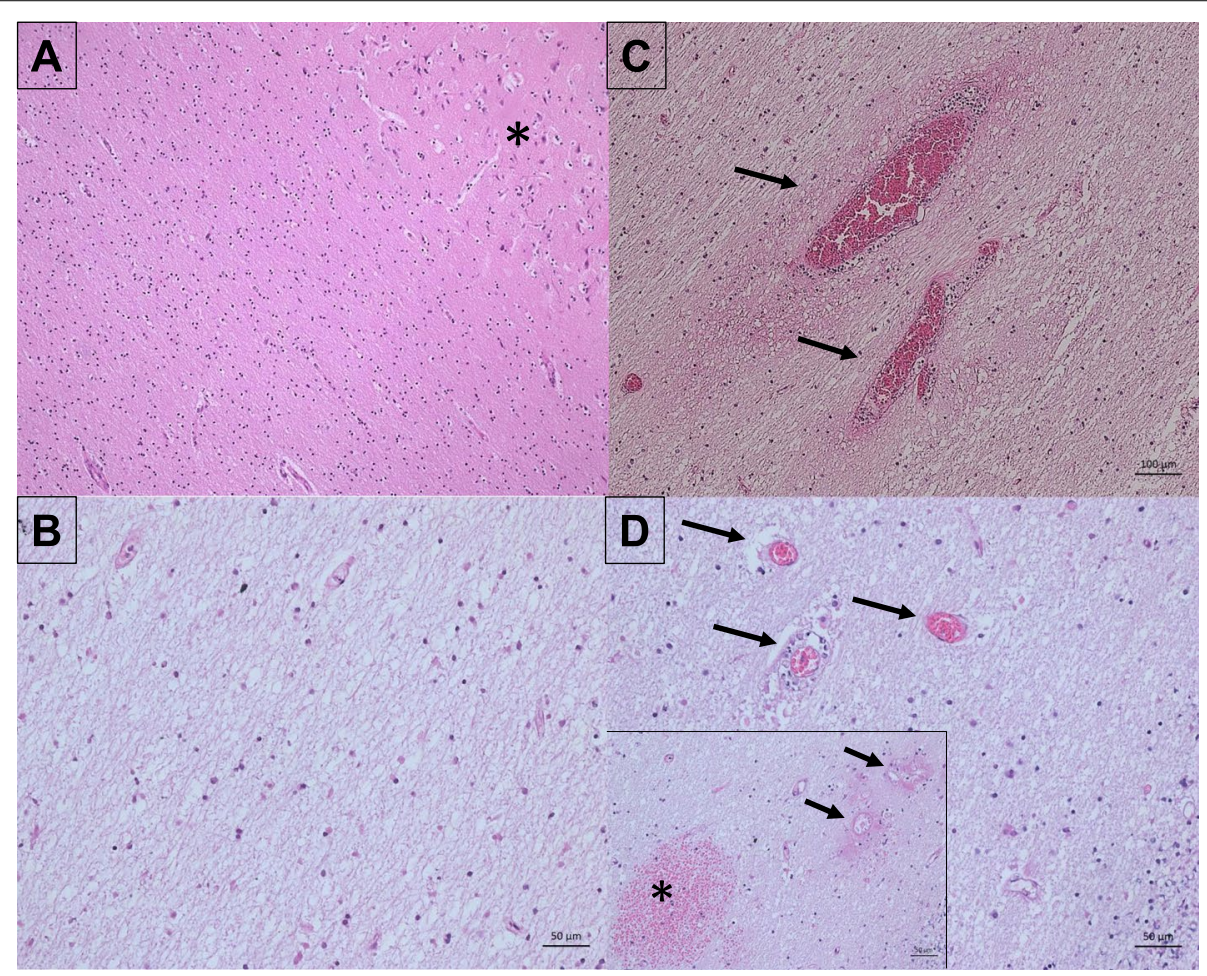

Fig. 2 Histopathological findings in a fatal case of PRES due to hypertension. A The frontal basal white matter appears normal. The black asterisk at the top right is to identify cerebral cortex with fresh hypoxemic-ischemic nerve cell damage in the form of cytoplasmic shrinkage, hypereosinophilia and nuclear condensation. B In contrast to frontal basal (A), the slices obtained from occipital white matter are characterized by diffuse myelin pallor and mildly increased glial cell density. C-D Slices obtained from cerebellar white matter with myelin pallor and mild reactive glial cell proliferation. In panel $\mathbf{C}$, one sees two thin-walled blood vessels with hyperemia and a perivascular exsudate indicative of endothelial cell damage (black arrows). Panel $\mathbf{D}$ shows three thin-walled hyperemic blood vessels (black arrows). The central blood vessel is surrounded by some macrophages with vacuolated cytoplasms ("phagocytes") indicative of myelin sheath break down products. In the small slice embedded in panel D, two thin-walled blood vessels (black arrows) with fibrinoid vascular wall necrosis can be seen at the top right and a petechial microbleeding area (black asterisk) at the bottom left. Hematoxylin and eosin staining of all slices; Scale bar $=100 \mu \mathrm{m} / 50 \mu \mathrm{m}$ 
symptoms. These findings are consistent with other study results $[1,5,9,10]$.

Interestingly, in our case series irreversibility was detected in $42 \%$ of patients in contrast to $18 \%$ in the Berlin PRES Study 2012 [1] and to $10-25 \%$ in the literature [5]. However, residual structural lesions including focal gliosis, infarction, posthemorrhagic residua, atrophy and laminar necrosis were seen in $43 \%$ of patients in the Berlin PRES Study [1]. There are some arguments that can explain the higher percentage of cases with nonreversible disease course $(42 \%)$ in our study. First, poor outcome of PRES seems related to a delayed diagnosis, thus supporting a previously report on delay in the elimination of the causes as an important predictor of poor outcome [11]. In addition, overrepresentation of critically ill patients $(80 \%$ of our patients required intensive care treatment) may be a potential indication of an irreversible course of PRES. Further, we followed the criteria proposed in the Berlin PRES Study 2012 for inclusion of the patients [1]. However, reversibility is not included in these criteria, because PRES is not always reversible and residual lesions may be encountered more frequently than commonly expected $[1,5]$.

In our study, PRES occurred in a wide range of disorders and predisposing conditions ranging from hypertension, eclampsia to stem cell transplantation, exposure to various immunosuppressants and cytostatic drugs as well as coproporphyria and serotonin syndrome. During the recent coronavirus disease 2019 (COVID-19) pandemic, COVID-19 infection has been identified as a further comorbid condition of PRES [12-14]. In one case, PRES showed a recurrence after a first episode 5 years before, suggesting a certain predisposition and the risk of recurrence if trigger factors are not eliminated, in this case hypertension. PRES can even involve the spinal cord, one patient with PRES in our study suffered in addition to brain lesions from spinal ischemia [2]. In this case, the spinal ischemia could be caused by PRES. In another case with Guillain-Barré syndrome in addition to PRES, we assume that the diseases occurred independently of each other, since PRES and Guillain-Barré syndrome have different pathomechanisms. CSF pleocytosis was found in $33 \%$ and elevated protein in $56 \%$ of our patients. We assumed the CSF pleocytosis as a result of a massive dysfunction of the blood brain barrier due to severe PRES, leading to increase of cell count following blood leakage. In two of these patients, subarachnoidal hemorrhage was detected. Other causes for CSF pleocytosis such as infection were excluded. In a previous study, all patients with CSF pleocytosis had atypical imaging features such as infarction or subarachnoidal hemorrhage [15]. In the literature, elevated total protein in CSF was reported to be correlated with severity of edema in PRES patients, whereas pleocytosis was rare [16], supporting the theory of a dysfunctional blood brain barrier.

Previous studies reported that vasoconstriction occurs in about $15-30 \%$ of patients with PRES who undergo angiography. Otherwise, PRES has also been reported in $17-38 \%$ of patients with reversible cerebral vasoconstriction syndrome (RCVS) [5]. Since pathophysiological mechanisms of PRES and RCVS are not completely known, it is still subject of debate whether PRES and RCVS are independent syndromes and sometimes overlap or are part of a continuum process [17]. In our patients, there was no evidence of vasoconstriction on vascular imaging including MR-angiography, CT-angiography, digital subtraction angiography (DSA) and extraand transcranial Doppler and Duplex sonography of brain-supplying arteries.

In our study population, mortality due to PRES was with $13 \%$ higher than the estimated 3-6\% in hospitalbased retrospective studies [5], indicating that early diagnosis, management of increased blood pressure and elimination of trigger factors are crucial for the outcome. However, another single center study reported a fatal outcome of $19,1 \%$ in their patients [18]. The histopathological findings in our patient with fatal course was consistent with previous findings [2, 6, 7] which confirm appearance of vasogenic and cytotoxic edema. Major histopathological findings included myelin pallor, prominent white matter vacuolization, swollen vascular endothelium, petechial hemorrhages, gliosis [2].

Limitation of our study is the small number of included patients and the retrospective nature. Further studies with a larger number of patients and a prospective study design are needed to confirm the cause-effect conclusions.

\section{Conclusions}

Our case series demonstrates a broad spectrum of clinical and neuroradiological manifestations of PRES, which was not in all cases reversible. Delay in diagnosis seems to contribute to limited reversibility and poor outcome. Histopathological findings in PRES are rare and can contribute to a better understanding of the pathophysiology.

\section{Abbreviations \\ CSF : Cerebral spinal fluid; EEG: Electroencephalography; mRS: modified Rankin Scale; PET-CT: Positron emission tomography-computed tomography; PRES: Posterior reversible encephalopathy syndrome; RCVS: Reversible cerebral vasoconstriction syndrome.}

\section{Acknowledgements \\ None.}

Authors' contributions

All named authors meet the International Committee of Medical Journal Editors (ICMJE) criteria for authorship of the manuscript, take responsibility 
for all aspects of the work in ensuring that questions related to the accuracy or integrity of any part of the work are appropriately investigated and resolved. FSI and IK contributed to the conception/design of the work. JN performed the histopathological studies. All authors contributed to analysis and interpretation of data for the study, FSI also to the acquisition of data. FSI and IK drafted the manuscript, JN revised it critically for important intellectual content. All authors have given final approval to the version to be published.

\section{Funding}

Open Access funding enabled and organized by Projekt DEAL.

\section{Availability of data and materials}

All data generated or analysed during this study are included in this published article. The data are available from the corresponding author upon request. No specific database was used for this case series, so public access to the database(s) is not applicable.

\section{Declarations}

\section{Ethics approval and consent to participate}

All procedures/protocols were in accordance with the ethic guidelines of the Ruhr University Bochum. All methods were carried out in accordance with relevant guidelines and regulations. According to legislative regulations in Germany, North Rhine-Westphalia and after consultation of the Institutional Review Board, Medical Faculty of the Ruhr University Bochum, the need for ethical approval and consent is deemed unnecessary for this retrospective case series with data collected during routine clinical work. Approval for brain autopsy was obtained.

\section{Consent for publication}

Not applicable.

\section{Competing interests}

The authors declare that they have no competing interests.

\section{Author details}

1 Department of Neurology, University Hospital Knappschaftskrankenhaus, Ruhr University Bochum, In der Schornau 23-25, 44892 Bochum, Germany.

${ }^{2}$ Institute of Pathology, Ruhr University Bochum, Bochum, Germany.

Received: 10 June 2021 Accepted: 14 September 2021

Published online: 06 October 2021

\section{References}

1. Liman TG, Bohner G, Heuschmann PU, Endres M, Siebert E. The clinical and radiological spectrum of posterior reversible encephalopathy syndrome: the retrospective Berlin PRES study. J Neurol. 2012;259:155-64.

2. Willard N, Honce JM, Kleinschmidt-DeMasters BK. PRES: review of histological features. J Neuropathol Exp Neurol. 2018;77:100-18.

3. Covarrubias DJ, Luetmer PH, Campeau NG. Posterior reversible encephalopathy syndrome: prognostic utility of quantitative diffusion-weighted MR images. AJNR Am J Neuroradiol. 2002;23:1038-48.
4. Sweany JM, Bartynski WS, Boardman JF. "Recurrent" posterior reversible encephalopathy syndrome: report of 3 cases-PRES can strike twice! J Comput Assist Tomogr. 2007;31:148-56.

5. Fugate JE, Rabinstein AA. Posterior reversible encephalopathy syndrome: clinical and radiological manifestations, pathophysiology, and outstanding questions. Lancet Neurol. 2015;14:914-25.

6. Kheir JN, Lawlor MW, Ahn ES, Lehmann L, Riviello JJ, Silvera VM, et al. Neuropathology of a fatal case of posterior reversible encephalopathy syndrome. Pediatr Dev Pathol. 2010;13:397-403.

7. Hayashi Y, Kimura A, Nakamura H, Mimuro M, Iwasaki Y, Hara A, et al. Neuropathological findings from an autopsied case showing posterior reversible encephalopathy syndrome-like neuroradiological findings associated with premedication including tacrolimus for autologous peripheral blood stem cell transplantation. J Neurol Sci. 2017;375:382-7.

8. Gao B, Lyu C, Lerner A, McKinney AM. Controversy of posterior reversible encephalopathy syndrome: what have we learnt in the last 20 years? J Neurol Neurosurg Psychiatry. 2018;89:14-20.

9. Bartynski WS, Boardman JF. Distinct imaging patterns and lesion distribution in posterior reversible encephalopathy syndrome. AJNR Am J Neuroradiol. 2007;28:1320-7.

10. Lee VH, Wijdicks EF, Manno EM, Rabinstein AA. Clinical spectrum of reversible posterior leukoencephalopathy syndrome. Arch Neurol. 2008;65:205-10.

11. Harirchian MH, Ghaffarpour M, Tabaeizadeh M, Siroos B. Immunosuppressive drugs, an emerging cause of posterior reversible encephalopathy syndrome: case series. J Stroke Cerebrovasc Dis. 2015;24:e191-195.

12. Gewirtz AN, Gao V, Parauda SC, Robbins MS. Posterior reversible encephalopathy syndrome. Curr Pain Headache Rep. 2021;25:19.

13. Parauda SC, Gao V, Gewirtz AN, Parikh NS, Merkler AE, Lantos J, White H, Leifer D, Navi BB, Segal AZ. Posterior reversible encephalopathy syndrome in patients with COVID-19. J Neurol Sci. 2020;416:117019.

14. Lallana S, Chen A, Requena M, Rubiera M, Sanchez A, Siegler JE, Muchada M. Posterior reversible encephalopathy syndrome (PRES) associated with COVID-19. J Clin Neurosci. 2021;88:108-12.

15. Ellis CA, McClelland AC, Mohan S, Kuo E, Kasner SE, Zhang C, Khankhanian P, Balu R. Cerebrospinal fluid in posterior reversible encephalopathy syndrome: implications of elevated protein and pleocytosis. Neurohospitalist. 2019:9:58-64.

16. Neeb L, Hoekstra J, Endres M, Siegerink B, Siebert E, Liman TG. Spectrum of cerebral spinal fluid findings in patients with posterior reversible encephalopathy syndrome. J Neurol. 2016;263:30-4.

17. Pilato F, Distefano M, Calandrelli R. Posterior reversible encephalopathy syndrome and reversible cerebral vasoconstriction syndrome: clinical and radiological considerations. Front Neurol. 2020;11:34

18. Alhilali LM, Reynolds AR, Fakhran S. A multi-disciplinary model of risk factors for fatal outcome in posterior reversible encephalopathy syndrome. Neurol Sci. 2014:347:59-65.

\section{Publisher's Note}

Springer Nature remains neutral with regard to jurisdictional claims in published maps and institutional affiliations.

Ready to submit your research? Choose BMC and benefit from:

- fast, convenient online submission

- thorough peer review by experienced researchers in your field

- rapid publication on acceptance

- support for research data, including large and complex data types

- gold Open Access which fosters wider collaboration and increased citations

- maximum visibility for your research: over $100 \mathrm{M}$ website views per year

At $\mathrm{BMC}$, research is always in progress.

Learn more biomedcentral.com/submissions 